\title{
Association of anemia with mobility capacity in older adults: a Korean nationwide population-based cross- sectional study
}

Ki Young Son ${ }^{1 *}$ (D) Dong Wook Shin ${ }^{2,3}$, Ji Eun Lee ${ }^{4}$, Sang Hyuck Kim ${ }^{5}$, Jae Moon Yun ${ }^{6,7}$ and Belong Cho $0^{6,7,8}$

\begin{abstract}
Background: Over $10 \%$ of adults aged $\geq 65$ years have anemia, as defined by the World Health Organization (WHO). As the timed up and go (TUG) test is one of the most widely used tests of mobility, this study investigated whether anemia was associated with mobility capacity assessed using the TUG test in older adults.

Methods: Subjects belonging to the Korean National Health Insurance Service-National Health Screening Cohort of the National Health Information Database were reviewed. Subjects were included if they had completed the TUG test as part of the National Screening Program for Transitional Ages in Korea. An abnormal TUG test result was defined as a time of $\geq 10 \mathrm{~s}$ and anemia was defined according to the WHO criteria as a hemoglobin $(\mathrm{Hb})$ concentration of $<13.0 \mathrm{~g} / \mathrm{dL}$ in men and $<12.0 \mathrm{~g} / \mathrm{dL}$ in women. The association between anemia and TUG test results was evaluated using four multiple logistic regression models with different levels of adjustment. Stratified analysis according to risk factors was performed.

Results: The 81,473 subjects included 41,063 (50.4\%) women and 40,410 (49.6\%) men. Mean TUG time was $8.44 \pm$ $3.08 \mathrm{~s}$, and abnormal TUG test results were observed in 22,138 (27.2\%) subjects. Mean Hb concentration was $13.72 \pm 1.41 \mathrm{~g} / \mathrm{dL}$, and 10,237 (12.6\%) subjects had anemia. U-shaped associations between $\mathrm{Hb}$ concentration and TUG test results were observed in both sexes. Subjects with anemia were 19\% more likely to have abnormal TUG test results, according to the fully adjusted model (adjusted odds ratio: 1.192, 95\% confidence interval: 1.137-1.247). Similar results were observed for both sexes. Stratified analysis showed that subjects with anemia were more likely to have abnormal TUG test results regardless of risk factors.

Conclusions: Individuals with anemia are more likely to have abnormal TUG test results, regardless of risk factors, than individuals without anemia. U-shaped relationships between $\mathrm{Hb}$ concentrations and TUG test results were observed in both sexes, although the optimal $\mathrm{Hb}$ concentration differed between men and women.
\end{abstract}

Keywords: Anemia, Timed up and go test, Older adults, Mobility capacity

\footnotetext{
*Correspondence: mdsky75@gmail.com

'Department of Family Medicine, Asan Medical Center, 88 Olympic-ro 43 gil, Songpa-gu, Seoul 05505, South Korea

Full list of author information is available at the end of the article
}

(C) The Author(s). 2020 Open Access This article is licensed under a Creative Commons Attribution 4.0 International License, which permits use, sharing, adaptation, distribution and reproduction in any medium or format, as long as you give appropriate credit to the original author(s) and the source, provide a link to the Creative Commons licence, and indicate if changes were made. The images or other third party material in this article are included in the article's Creative Commons licence, unless indicated otherwise in a credit line to the material. If material is not included in the article's Creative Commons licence and your intended use is not permitted by statutory regulation or exceeds the permitted use, you will need to obtain permission directly from the copyright holder. To view a copy of this licence, visit http://creativecommons.org/licenses/by/4.0/ The Creative Commons Public Domain Dedication waiver (http://creativecommons.org/publicdomain/zero/1.0/) applies to the data made available in this article, unless otherwise stated in a credit line to the data. 


\section{Background}

Anemia is a common condition in older adults and is due to multiple factors. Over $10 \%$ of adults aged $\geq 65$ years have anemia, as defined by the World Health Organization (WHO) [1]. Anemia is caused by nutritional deficiency in one third of these individuals, by chronic illness, including renal insufficiency, in another third, and by undetermined factors in another third [1-3]. Anemia in older adults is associated with various negative health outcomes, including reduced quality of life [3-5], depressive mood and fatigue $[4,5]$, falls $[6,7]$, frailty $[3,8]$, impairment in ADL [4, 9-11], cognitive impairment [10], and increased mortality $[12,13]$.

Anemia may reduce muscle oxygenation, consequently affecting muscle strength and quality and, therefore, physical performance including mobility capacity [14, 15]. Given the importance of physical performance for health outcomes and quality of life in older adults, and the fact that anemia is both common in older adults and easily discovered in the usual primary care setting, it is important to examine the association between anemia and physical performance. Studies assessing the association between functional decline in mobility and anemia in older adults have shown that anemia is associated with impaired standing balance $[4,14,15]$, slower gait speed $[4,14-16]$, slower chair standing $[4,14,15]$, decreased muscle strength including, handgrip and knee extensor strengths [4, 15], and multidimensional loss of function [17].

The timed up and go (TUG) test is one of the most widely used tests of mobility capacity in older adults. It includes standing and walking activities commonly performed in daily life, including walking, turning, and transitions [18]. The test is easy to perform in clinical setting, and is useful to assess aspects of mobility, such as static/dynamic balance, lower extremities strength, and gait speed. Previous studies reported that TUG test predicted falls, fractures, hospital admissions due to fractures [19], disability [20], low quality of life [21], low social participation [21], complications after elective surgery in patients with cancer [22], and onset of difficulty in activities of daily living (ADL) [23].

In contrast, previous studies assessing the association between anemia and TUG test results as a mobility measure have yielded inconsistent results, with some reporting an association between anemia and impaired TUG test results (i.e., $0.14-0.36 \mathrm{~s}$ improvement in TUG test results with a $1 \mathrm{mg} / \mathrm{dL}$ increase in $\mathrm{Hb} ; 1.5$ times greater proportion of impaired TUG test results in anemic group) $[16,24]$, and others reporting no association $[21,25]$. These studies, however, included relatively few subjects (i.e., 62, 93, 122, and 236 participants, respectively) and were limited to specific populations such as patients with hip arthroplasty or patients with anemia treated with epoetin alfa. To our knowledge, no study to date has assessed these relationships in large general populations including sufficient numbers of people with anemia.

The present study therefore evaluated the association between anemia and mobility capacity assessed using the TUG test in a large general population of adults aged 66 years who had been enrolled in the National Screening Program for Transitional Ages (NSPTA) in Korea, a nationwide representative sample of Korean individuals. We hypothesized that anemia in older adults would be associated with an increased likelihood of impaired TUG test results.

\section{Methods}

\section{Study design}

This study was a population-based cross-sectional study using the National Health Information Database (NHID) in Korea. The participants were 66-year-old men and women who underwent the NSPTA examination between 2007 and 2015. All assessments were performed on the day of the NSPTA examination. We aimed to compare TUG test results between normal and anemic groups using multiple logistic regression analysis.

\section{Data sources}

The Korean National Health Insurance Service (KNHIS) is a publicly available health insurance plan that provides universal health coverage to almost all Koreans except Medicaid beneficiaries, who account for $<3 \%$ of the population. The KNHIS is responsible for the National Screening Program (NSP), a biennial health screening program. KNHIS added the NSPTA to the NSP in 2007. The purposes of addition of the NSPTA are to tailor the NSP by the age and sex of participants and to strengthen counseling after examination. Only participants aged 66 years of the NSPTA undergo TUG tests and unipedal stance tests to assess mobility capacity.

The KNHIS created the NHID. The NHID includes healthcare utilization data, the NSPTA health examination results, sociodemographic information, and mortality data of over 50 million persons in Korea [26]. The details of the NSPTA have been described elsewhere [27].

\section{Study population}

The National Health Insurance Service-National Health Screening Cohort (NHIS-HEALS) database of the NHID included 515,867 randomly selected subjects, representing approximately $10 \%$ of Korean participants aged $40-$ 79 years who participated in the NSP at least once in 2002-2003. Subjects aged 66 years who underwent the NSPTA examination between 2007 and 2015 were included. We excluded participants with disabilities at baseline (i.e., registered persons with disabilities and those with disabilities in daily activities). After excluding 
subjects who were registered as persons with disabilities in the Korean National Disability Registry (KNDR), those with missing TUG test results, and those with impairments in ADL, 81,473 subjects were included (Fig. 1).

The database included the participants' demographic characteristics, previous medical history and health behaviors, and screening test results. Factors recorded included height, weight, abdominal circumference, and the results of physical function tests (i.e., TUG and unipedal stance tests) and laboratory tests.

The study protocol was approved by the Institutional Review Board of Seoul National University Hospital (IRB No. E-1703-020-836), which waived the requirement for informed consent because the KNHIS database was constructed after anonymization according to strict confidentiality guidelines. We, authors acquired permission to access the database by National Health Insurance Sharing Service (NHIS-2018-2-201). And this study used the database of NHID constructed by KNHIS, with the permission. In this reason, this study is not registered separately to clinical trial registry.

\section{Variables}

Independent variables

Anemia Anemia and hemoglobin $(\mathrm{Hb})$ concentration were the independent variables in this study. Anemia was defined according to the WHO criteria as an $\mathrm{Hb}$ concentration of $<13.0 \mathrm{~g} / \mathrm{dL}$ in men and $<12.0 \mathrm{~g} / \mathrm{dL}$ in women [28], based on laboratory test results recorded by the NSPTA. The optimal $\mathrm{Hb}$ concentration associated with the fastest TUG time was determined by assessing $\mathrm{Hb}$ concentration as a continuous variable.

\section{Outcome variable}

Mobility capacity The TUG test result was the outcome variable in this study, representing mobility capacity in study participants. Each subject performed the TUG test on the day of physical examination of the NSPTA, in the clinical setting of each subject's community hospital according to the NSPTA manual. Participants were instructed to sit on a chair (i.e., a normal clinic chair without armrests), stand, walk $3 \mathrm{~m}$ at a comfortable speed, walk back to the chair, and sit again on the chair, all while wearing regular footwear and/or using walking aids. The time from standing to sitting again was measured once, and a time longer than $10 \mathrm{~s}$ was categorized as abnormal. According to the NSPTA manual, a TUG test result of $>10 \mathrm{~s}$ is regarded as requiring attention, and a TUG test result of $>20 \mathrm{~s}$ is regarded as impaired. However, a previous study using NSPTA data showed that only a small number of participants were classified as impaired when $20 \mathrm{~s}$ was used as the TUG test result threshold for 66-year-olds in Korea [2]. For this reason, we used $10 \mathrm{~s}$ as our threshold. The details of how the TUG test is conducted in the NSPTA have been described elsewhere [29].

\section{Potential confounding variables}

In our analysis, we included potential confounders that had been shown to affect physical performance in previous studies, and for which data were available in the database, such as chronic diseases, obesity, cognitive impairment, and depression. Data on chronic diseases, such as hypertension, diabetes mellitus, and dyslipidemia, were collected from the questionnaire and laboratory

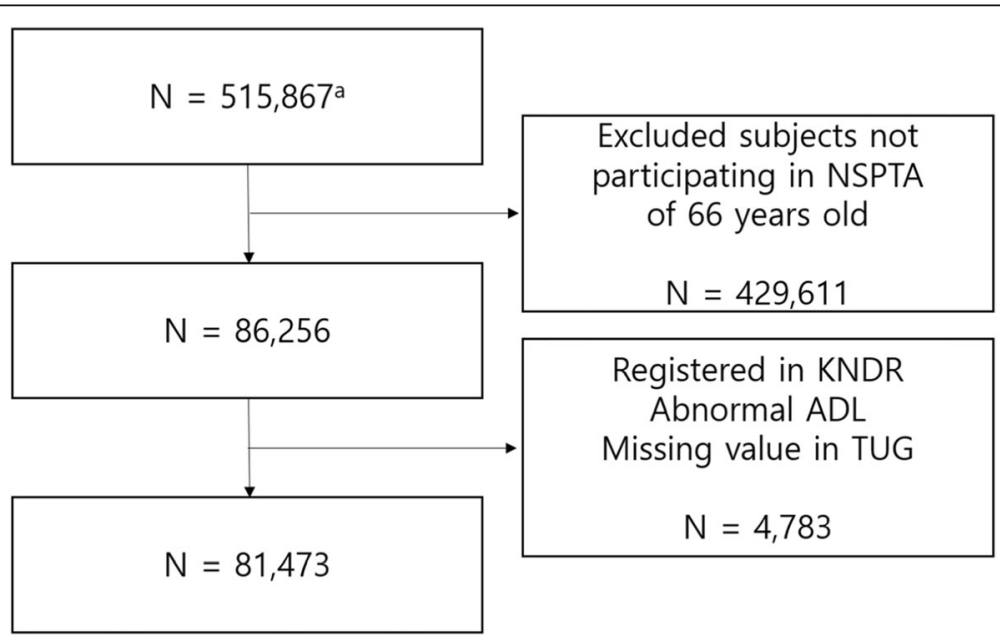

Fig. 1 Flow of study subjects. a. Subjects of the National Health Insurance Service-National Health Screening Cohort database of the Korean National Health Insurance Service (2002-2015). *Abbreviation: NSPTA, National Screening Program for Transitional Ages in Korea; KNDR, Korean National Disability Registry; ADL, Activity of daily living; TUG, Timed up and go 
results of the NSPTA. Each condition was defined as previously described [30]. Subjects who took medications for hypertension, diabetes mellitus, or dyslipidemia were considered to have the corresponding disease. In addition, hypertension was defined as a systolic blood pressure (SBP) of $\geq 140 \mathrm{mmHg}$ or a diastolic blood pressure (DBP) of $\geq 90 \mathrm{mmHg}$; diabetes mellitus as a fasting serum glucose concentration of $\geq 126 \mathrm{mg} / \mathrm{dL}$; and dyslipidemia as a serum total cholesterol (TC) concentration of $\geq 240 \mathrm{mg} / \mathrm{dL}$.

Body mass index (BMI), cognitive impairment, and ADL were determined as previously described [29]. BMI was calculated as weight divided by height squared $\left(\mathrm{kg} / \mathrm{m}^{2}\right)$. Normal BMI was defined as between 18.5 and $23 \mathrm{~kg} / \mathrm{m}^{2}$ and obesity was defined as $\geq 25 \mathrm{~kg} / \mathrm{m}^{2}$ according to Asianspecific criteria.

Cognition was assessed using the Korean Dementia Screening Questionnaire-Cognition (KDSQ-C). The KDSQ-C is a self-administered, consisting of 15 items, each rated on a three-point Likert scale $(0,1$, or 2 , with a higher score considered worse). When participants' composite score $\geq 6$, they were considered as cognitively impaired. The KDSQ-C is validated questionnaire [31] and integrated in the NSPTA questionnaire.

The NSPTA questionnaire included six ADL items, which derived from the Korean versions of the ADL (K$\mathrm{ADL}$ ) and Instrumental ADL (K-IADL) questionnaires [32]. Following four items derived from the K-ADL: "Do you bathe by yourself without help?," "Do you dress by yourself without help?," "Do you eat by yourself without help if a meal is prepared?," and "Do you go to the toilet by yourself without help?" Following two items derived from the K-IADL: "Do you prepare your own meals by yourself without help?" and "Do you go outside by yourself to places within walking distance?" If participants answered "No" to one or more of these questions, they were classified as having impairments in ADL.

Three questions, which derived from the validated Korean version of the Geriatric Depression Scale, were used to assess depressive mood [33]. The questions were: "Do you feel that your activity or desire has decreased recently?," "Do you feel that you are currently useless?," and "Do you feel that you are currently hopeless?" If participants answered "Yes" to any of these questions, they were categorized as depressed.

\section{Statistical analysis}

Baseline characteristics are expressed as frequencies and percentages. Continuous variables are reported as mean \pm standard deviation (SD), and categorical variables as frequencies and percentages.

Multiple logistic regression models were used to evaluate the association between TUG test results and anemia. In these analyses, all variables were expressed as categorical variables as defined above. Four models were built for these analyses: a crude model and three adjusted models. Model 1 was adjusted for sex; Model 2 was adjusted for sex, depressive mood, and cognitive impairment; and Model 3 was adjusted for all the factors in Model 2, as well as BMI, chronic diseases (i.e., hypertension, diabetes mellitus, dyslipidemia), and creatinine concentration. Odds ratios (OR) and $95 \%$ confidence intervals (CI) were calculated for each model.

Participants with abnormal TUG test results thought to indicate risk were identified by stratified analyses in Model 3, with participants stratified by sex, depressive mood, cognitive impairment, obesity, chronic diseases, and cardiovascular diseases.

In addition, we fitted fractional polynomial prediction plots with $95 \%$ CI to demonstrate the prediction of TUG test results according to $\mathrm{Hb}$ concentrations, expressed as continuous variables.

Statistical analyses were performed using Stata software (version 15.1; StataCorp, College Station, Texas). A $P$-value of $<0.05$ was considered significant.

\section{Results}

\section{Baseline characteristics of participants}

The NHIS-HEALS database included 515,867 subjects. Of the potentially eligible participants, we excluded 429 , 611 participants who did not participate in the NSPTA for 66-year-olds. Of the remaining 86,256 participants, we excluded those with disabilities at baseline, those who were registered in the KNDR $(n=1255)$, and those with impairments in ADL $(n=3448)$. In addition, of the remaining 81,531 participants, we excluded those who had missing TUG test results $(n=58)$. Ultimately, 81,473 subjects were included (Fig. 1).

These 81,473 subjects included 41,063 (50.4\%) women and 40,410 (49.6\%) men. Mean TUG time was $8.44 \pm$ $3.08 \mathrm{~s}$, with $22,138(27.2 \%)$ subjects having abnormal TUG test results $(\geq 10 \mathrm{~s})$. All participants in this study were aged 66 years, because only 66-year-olds underwent the TUG test in the NSPTA. Mean Hb concentration was $13.72 \pm 1.41 \mathrm{~g} / \mathrm{dL}$, with 10,237 (12.6\%) participants having anemia. Mean BMI was $24.2 \pm 3.0 \mathrm{~kg} / \mathrm{m}^{2}$, with $1678(2.1 \%)$ subjects being underweight $(\mathrm{BMI}<18.5 \mathrm{~kg} /$ $\left.\mathrm{m}^{2}\right)$ and $32,143(37.0 \%)$ being obese (BMI $\left.\geq 25.0 \mathrm{~kg} / \mathrm{m}^{2}\right)$.

Approximately $20 \%$ of participants reported having depressive mood and 15\% were cognitively impaired. Approximately $70 \%$ had hypertension, while approximately 26 and $28 \%$ had diabetes and dyslipidemia, respectively. Only $0.8 \%$ had cardiovascular disease (Table 1 ).

\section{Association between anemia and timed up and go test results}

$\mathrm{U}$-shaped associations between $\mathrm{Hb}$ concentration and TUG test results were observed in both sexes. Optimal 
Table 1 Baseline characteristics of study subjects

\begin{tabular}{|c|c|c|c|}
\hline & \multirow[t]{2}{*}{ Total } & \multicolumn{2}{|c|}{ Timed up and go results } \\
\hline & & Normal & Abnormal \\
\hline & N (\%) & N (\%) & N (\%) \\
\hline Sex (female) & $41,063(50.4)$ & $28,941(48.8)$ & $12,122(54.8)$ \\
\hline Timed up and go test result (s) & $8.44 \pm 3.08$ & $7.20 \pm 1.49$ & $11.76 \pm 3.73$ \\
\hline Hemoglobin $(\mathrm{g} / \mathrm{dL})^{*}$ & $13.72 \pm 1.41$ & $13.77 \pm 1.40$ & $13.58 \pm 1.43$ \\
\hline Anemia & $10,237(12.6)$ & $7086(11.9)$ & $3151(14.2)$ \\
\hline \multicolumn{4}{|l|}{ BMI $\left(\mathrm{kg} / \mathrm{m}^{2}\right)^{* *}$} \\
\hline Underweight (< 18.5) & $1678(2.1)$ & $1211(2.0)$ & $467(2.1)$ \\
\hline Normal weight (18.5-25) & $49,652(60.9)$ & $36,546(61.6)$ & $13,106(59.2)$ \\
\hline Overweight ( $\geq 25$ ) & $32,143(37.0)$ & $21,578(36.4)$ & 8565 (38.7) \\
\hline Depressive mood & $16,458(20.3)$ & $11,342(19.2)$ & $5116(23.2)$ \\
\hline Cognitive impairment & $12,247(15.1)$ & $8488(14.4)$ & $3759(17.1)$ \\
\hline Hypertension & $58,695(71.7)$ & $42,023(70.8)$ & $16,372(74.0)$ \\
\hline Diabetes mellitus & $21,065(25.9)$ & $14,638(24.7)$ & $6427(29.0)$ \\
\hline Dyslipidemia & $23,026(28.3)$ & $16,149(27.2)$ & $6877(31.1)$ \\
\hline Cardiovascular disease & $649(0.8)$ & $484(0.8)$ & $165(0.8)$ \\
\hline
\end{tabular}

Results are reported as mean \pm standard deviation or as number (\%).

${ }^{*}$ Anemia was defined as a hemoglobin concentration of $<13 \mathrm{~g} / \mathrm{dL}$ in men and $<12 \mathrm{~g} / \mathrm{dL}$ in women

**Abbreviation: $B M I$ Body mass index

TUG test results were obtained at approximately 16.0-17.0 $\mathrm{g} / \mathrm{dL} \mathrm{Hb}$ in men, and at approximately $13.0-15.0 \mathrm{~g} / \mathrm{dL}$ in women (Fig. 2).

Of the 71,236 subjects with non-anemic Hb concentrations, 18,987 (26.7\%) had abnormal TUG test results, compared with 3151 (30.8\%) of the 10,237 subjects with anemia.

People with anemia were $22 \%$ more likely to have abnormal TUG test results than people without anemia, according to the crude model (OR: 1.224, 95\% CI: $1.170-1.280$ ), and were $19 \%$ more likely to have abnormal TUG test results according to the fully adjusted model (adjusted OR (aOR): 1.192, 95\% CI: 1.137-1.247).

Similar results were found in both sexes. Anemic men were 16\% more likely (aOR: 1.160, 95\% CI: 1.078-1.249) and anemic women were $21 \%$ more likely (aOR: 1.210 , 95\% CI: $1.141-1.284)$ to have abnormal TUG test results, according to the fully adjusted model (Table 2).

\section{Stratified analysis}

Stratified analysis showed that participants with anemia were more likely to have abnormal TUG test results regardless of risk factors (e.g., sex, depressive mood, cognitive impairment, obesity, and chronic diseases), except for participants with cardiovascular disease (Fig. 3).

\section{Discussion}

This large general-population-based study evaluated the association between anemia and TUG test results in a nationally representative sample of 66-year-old subjects in an Asian country. The use of a fully adjusted model showed that anemia was associated with abnormal TUG test results and that $\mathrm{Hb}$ concentrations and TUG test results had a U-shaped relationship, with the $\mathrm{Hb}$ concentration associated with optimal TUG test results differing between men and women. Stratified analysis found that the association between anemia and TUG was independent of risk factors, including sex, depressive mood, impaired cognition, obesity, and chronic diseases.

A previous study of 100 inpatients aged $\geq 70$ years reported that anemia and low $\mathrm{Hb}$ level were associated with multidimensional loss of function, in particular a 2.32-times higher likelihood of abnormal TUG test results [23]. However, studies assessing the association between anemia and TUG test results have yielded inconsistent results. For example, a community-based study in Iceland found that a $1 \mathrm{mg} / \mathrm{dL}$ increase in $\mathrm{Hb}$ concentration was associated with a 0.14 -to-0.36 s improvement in TUG test results and predicted response to 12 -week resistance exercise [22]. Similarly, a study of 93 community-dwelling older adults in Egypt found that anemia was associated with increased rates of abnormal TUG test results (64.3\% of participants with anemia vs. $32.9 \%$ of participants without anemia) [24]. However, treatment of chronic anemia with epoetin alfa increased $\mathrm{Hb}$ concentration by $2 \mathrm{~g} / \mathrm{dL}$ and improved fatigue and subject quality of life, but did not improve TUG test results [11]. Similarly, a study of patients who underwent hip arthroplasty reported that differences in $\mathrm{Hb}$ concentration from before to after surgery were not associated 
A.

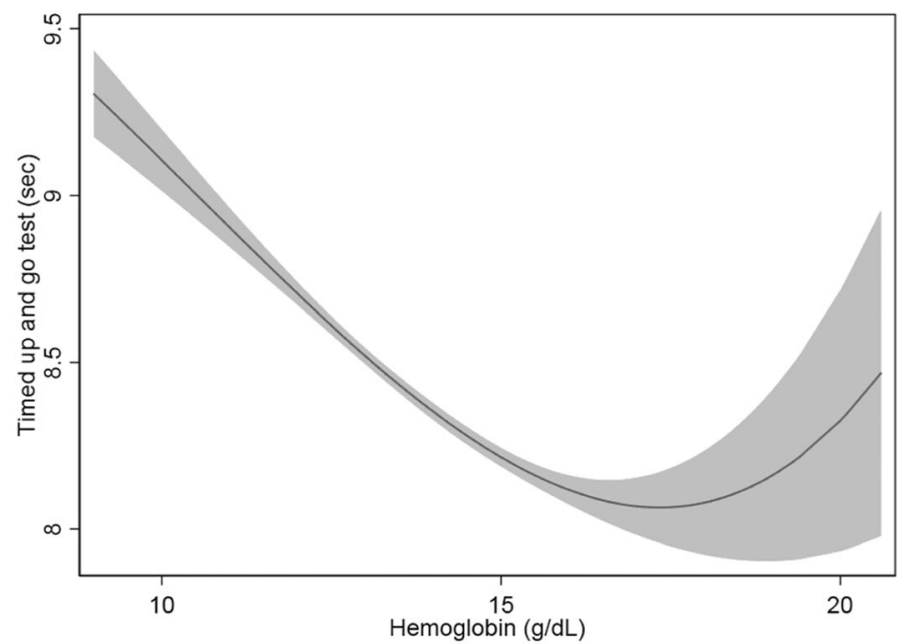

B.

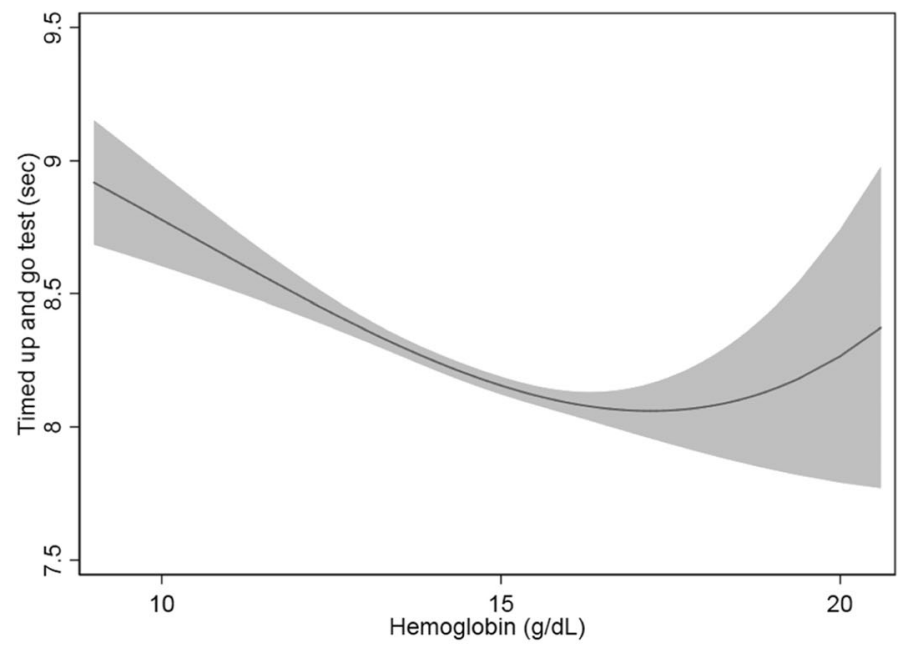

C.

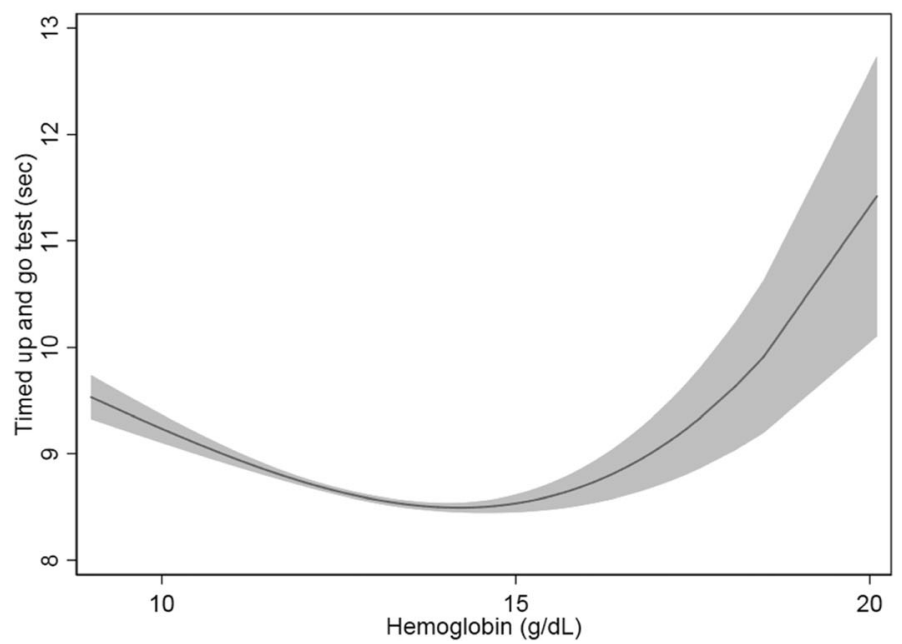

Fig. 2 Fractional polynomial prediction plots with 95\% confidence interval of association of timed up and go test results with hemoglobin concentration in a) all participants, b) men, and c) women. Gray areas depict $95 \%$ confidence intervals 
Table 2 Associations between anemia and timed up and go test results

\begin{tabular}{|c|c|c|c|c|c|c|c|}
\hline & Anemia & $\begin{array}{l}\text { Total } \\
\text { (N) }\end{array}$ & $\begin{array}{l}\text { Abnormal TUG** } \\
\text { Result (N) }\end{array}$ & $\begin{array}{l}\text { Crude } \\
\text { OR* }^{* *}(95 \% \mathrm{Cl})\end{array}$ & $\begin{array}{l}\text { Model } 1 * \\
\text { aOR }^{* *}\left(95 \% \mathrm{Cl}^{* *}\right)\end{array}$ & $\begin{array}{l}\text { Model } 2 * \\
\text { aOR }(95 \% \mathrm{Cl})\end{array}$ & $\begin{array}{l}\text { Model 3* } \\
\text { aOR }(95 \% \mathrm{Cl})\end{array}$ \\
\hline \multirow[t]{3}{*}{ Total } & All & 81,473 & 22,138 & & & & \\
\hline & Normal & 71,236 & 18,987 & Ref & Ref & Ref & Ref \\
\hline & Anemic & 10,237 & 3151 & $1.224(1.170-1.280)$ & $1.195(1.142-1.250)$ & $1.188(1.135-1.243)$ & $1.192(1.137-1.247)$ \\
\hline \multicolumn{8}{|l|}{ Sex } \\
\hline \multirow[t]{3}{*}{ Male } & All & 40,410 & 10,016 & & & & \\
\hline & Normal & 36,290 & 8876 & Ref & N/A & Ref & Ref \\
\hline & Anemic & 4120 & 1140 & $1.182(1.099-1.270)$ & & $1.169(1.087-1.257)$ & $1.160(1.078-1.249)$ \\
\hline \multirow[t]{3}{*}{ Female } & All & 41,063 & 12,122 & & & & \\
\hline & Normal & 34,946 & 10,111 & Ref & N/A & Ref & Ref \\
\hline & Anemic & 6117 & 2011 & $1.203(1.135-1.275)$ & & $1.200(1.132-1.272)$ & $1.210(1.141-1.284)$ \\
\hline
\end{tabular}

*Model 1: Crude model + adjustment for sex

Model 2: Model 1 + adjustment for depressive mood and cognitive impairment.

Model 3: Model $2+$ adjustment for BMI, hypertension, diabetes mellitus, dyslipidemia, and creatinine level.

**Abbreviations: TUG Timed up and go test, OR Odds ratio, aOR Adjusted odds ratio, CI Confidence interval, N/A Not applicable

with TUG test results [25]. These previous studies, however, included relatively few subjects and were limited to specific populations, whereas the present study used a large, nationally representative sample of Asian adults aged 66 years. Therefore, this study provides evidence to overcome the limited generalizability of the relationship between anemia and TUG test results, especially in general populations of young-old individuals. However, because the present study was cross-sectional in design, its results do not indicate a causal relationship between anemia and TUG. Hence, large longitudinal studies are needed to evaluate this association.

In the present study, $\mathrm{Hb}$ concentrations and TUG test results were found to have a U-shaped relationship, with the best TUG test results obtained at approximately $16.0-17.0 \mathrm{~g} / \mathrm{dL} \mathrm{Hb}$ in men, and at approximately 13.0 $15.0 \mathrm{~g} / \mathrm{dL}$ in women. Because normal $\mathrm{Hb}$ concentration ranges are $14-18 \mathrm{~g} / \mathrm{dL}$ in men and $12-16 \mathrm{~g} / \mathrm{dL}$ in women

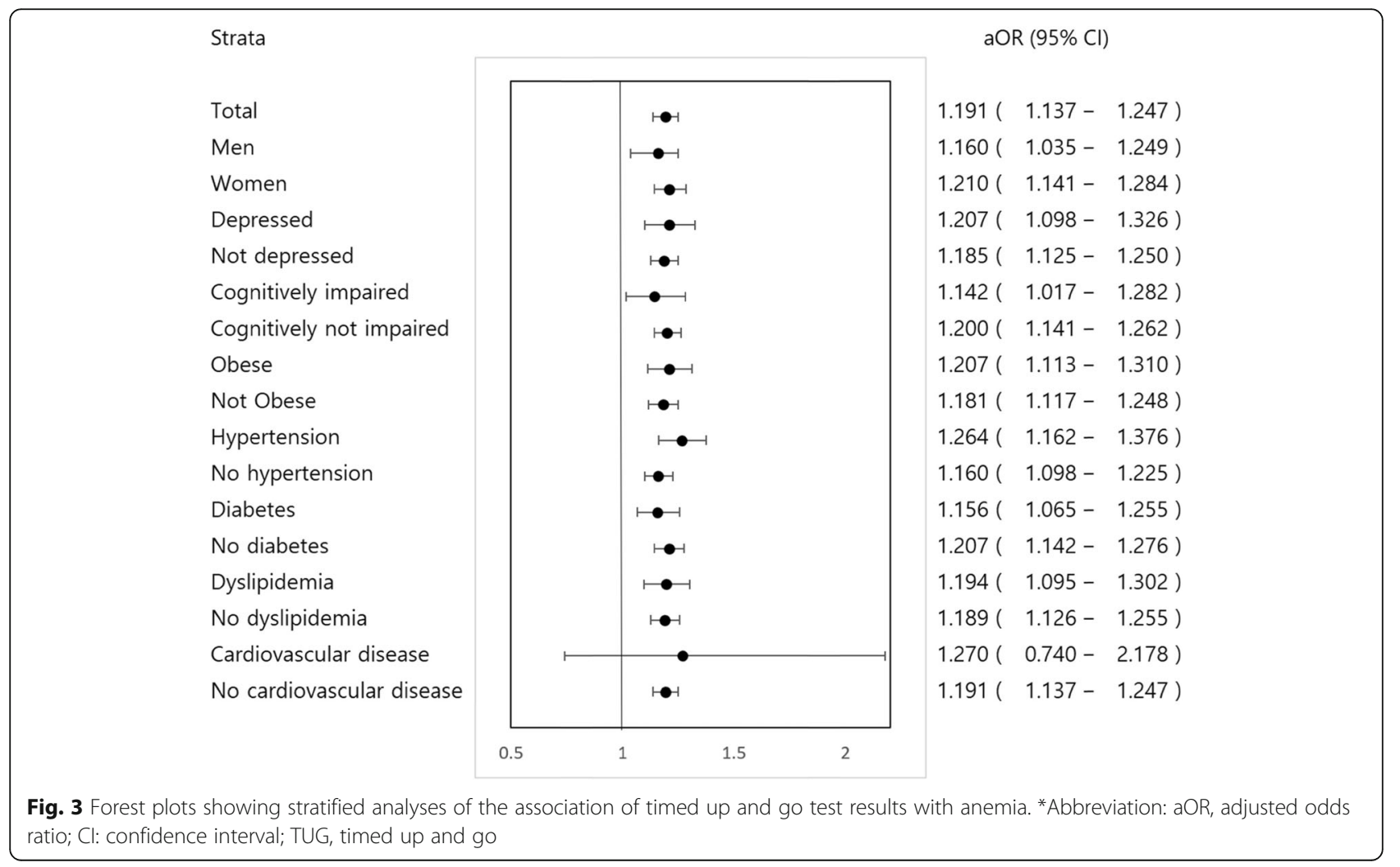


according to the WHO criteria [28], these results indicate that the optimal range of $\mathrm{Hb}$ concentration for mobility capacity, determined using the TUG test results, is higher than the lower limit of normal $\mathrm{Hb}$ concentrations. This finding is consistent with a previous study showing that anemia is associated with mobility difficulty in community-dwelling women [34]. A Ushaped relationship was also observed between $\mathrm{Hb}$ concentration and mobility in the aforementioned study, with mobility difficulty being significantly lower at an $\mathrm{Hb}$ concentration of $13.5 \mathrm{~g} / \mathrm{dL}$ than at $12.0 \mathrm{~g} / \mathrm{dL}$ [34].

Owing to the cross-sectional design of this study, it was not possible to evaluate whether the association between anemia and TUG test results was causative. Anemia may not be an independent risk factor, but rather a marker for other risk factors for reduced physical performance, such as comorbid chronic diseases. However, a stratified analysis found that the association between anemia and TUG test results was robust regardless of risk factors, except for cardiovascular and cerebrovascular diseases, suggesting that anemia may be independently associated with TUG test results. This finding suggests that caution should be exercised regarding possible physical performance deterioration when a physician identifies an older adult with anemia or low-normal $\mathrm{Hb}$ concentration, which is a commonly identified condition in a primary care setting.

The exact mechanism by which anemia negatively affects physical performance remains incompletely understood. Anemia may reduce muscle oxygenation, consequently affecting muscle strength and quality and, therefore, physical performance. This hypothesis has been applied to the association between other vascular conditions causing muscular hypoperfusion (i.e., diabetes mellitus, peripheral vascular disease, and cardiovascular disease) and reduced physical performance in older adults. Alternatively, increased chronic inflammation may result in a greater decline in physical performance [35]. This hypothesis is supported by the identification of increased C-reactive protein concentrations in older adults, observed in another study population [36]. Additional studies are required to determine the mechanism linking anemia and physical performance. Furthermore, there appears to be a general optimal range of $\mathrm{Hb}$ concentration for physical performance, which means that no proportional enhancement of performance is observed above a certain concentration of $\mathrm{Hb}$. This is supported by the findings of a study involving elite athletes, in which $\mathrm{Hb}$ concentration was not titrated toward the upper allowed limit for performance enhancement [37]. This may explain the $\mathrm{U}$-shaped relationship between $\mathrm{Hb}$ concentration and TUG test results.

Much like studies assessing the association between anemia and TUG test results, studies assessing the association between anemia or low $\mathrm{Hb}$ level and falls have yielded inconsistent results. A prospective general US population-based study reported that low $\mathrm{Hb}$ concentration was a risk factor for recurrent falls [13], whereas two German studies reported no association between anemia and risk of falling [12, 38]. Because falls are an important health outcome in older adults and the TUG test is regarded as useful in assessing fall risk, larger longitudinal studies are required to confirm the association between anemia and falls, including the role of the TUG test in this association.

Because participants in this study participated in the NSP voluntarily, there is a possibility of selection bias, in that participants were healthy enough to travel to the NSP facility and concerned enough about their health to do so, perhaps more so on both counts than non-participants. We cannot examine the differences between participants and non-participants in the NSP because there is a lack of data on non-participants in the program. However, it is not probable that this bias affects potential participants differently according to their anemia status, because anemia is not among the factors affecting participation in the NSP in this relatively healthy population after excluding people with disabilities. Furthermore, although participants with TUG test results had increased rates of diabetes mellitus, dyslipidemia, and depressive mood (data not shown), compared with participants of the same age who did not have TUG test results, there were no evident differences in $\mathrm{Hb}$ concentration or rate of anemia between these two groups $(13.7 \mathrm{~g} / \mathrm{dL}$ vs. $13.7 \mathrm{~g} / \mathrm{dL}$, and $12.5 \%$ vs. $11.2 \%$, respectively).

This study had several limitations, including its crosssectional design, which prevented us from answering some of the questions that we have discussed, based on the results of this study. For this reason, we have only speculated about the answers to these questions, based on the findings of this study and previous studies. Longitudinal studies are needed to evaluate this association. In addition, the study population included only 66-year-old Korean men and women, preventing assessment of the association between TUG test results and outcomes in different age and ethnic groups. However, this large general-population study on the association between anemia and TUG test results overcame the limitation of generalizability present in previous studies involving small numbers of participants and specific populations. In addition, because the TUG test was performed in a clinical setting according to the NSPTA manual, there were procedural variations in the TUG test measurements, such as the type of chair used. Moreover, cut-off value $(10 \mathrm{~s})$ for TUG in NSPTA manual is not strictly based on scientific data, but on expert consensus in Korea. There are many different possible cut-off values for TUG (e.g. $12 \mathrm{~s}, 15 \mathrm{~s}$ ), and the effect size or association 
could be altered if we used different cut-off value. When we applied $12 \mathrm{~s}$ and $15 \mathrm{~s}$ as cut-off in Model 3, we found aORs were 1.268 (95\% CI: $1.192-1.285)$ and 1.204 (95\% CI: 1.110-1.308), respectively. (Data not shown) The limitation of the database prevented adjustment for risk factors such as education and dietary intake. In addition, although there is no single consensus on the threshold value for the TUG test, we used $10 \mathrm{~s}$ as the threshold of impairment because participants were young-old and only a small number $(730 / 81,473)$ of participants were classified as impaired when $20 \mathrm{~s}$ was used as the threshold. It may be arguable whether $10 \mathrm{~s}$ is the best threshold for this population. Lastly, because the NHID is fundamentally a database for medical claims, some diagnoses may have been misclassified. However, every claim in this dataset was audited by the Korean Health Insurance Review and Assessment before payment, making the misclassification of diagnoses improbable.

\section{Conclusions}

Individuals with anemia are more likely to have abnormal TUG test results than individuals without anemia, regardless of risk factors. Hb concentrations and TUG test results have a U-shaped relationship, although optimal $\mathrm{Hb}$ concentrations differ between men and women.

\section{Abbreviations \\ ADL: Activities of daily living; aOR: Adjusted odds ratio; BMI: Body mass index; Cl: Confidence interval; DBP: Diastolic blood pressure; Hb: Hemoglobin; IADL: Instrumental activities of daily living; KDSQ-C: Korean Dementia Screening Questionnaire-Cognition; KNDR: Korean National Disability Registry; KNHIS: Korean National Health Insurance Service: NHID: National Health Information Database; NHIS-HEALS: National Health Insurance Service-National Health Screening Cohort; NSP: National Screening Program; NSPTA: National Screening Program for Transitional Ages; OR: Odds ratio; SBP: Systolic blood pressure; SD: Standard deviation; TC: Total cholesterol; TUG: Timed Up and Go; WHO: World Health Organization}

\section{Acknowledgments}

Not applicable.

\section{Authors' contributions}

Study concept and design: KYS, DWS, BLC, JEL. Acquisition of data: KYS, DWS, JMY, SHK. Analysis and interpretation of data: KYS. Drafting of manuscript: KYS. Critical revision of the manuscript: JMY, SHK, JEL. All authors read and approved the final manuscript.

\section{Funding}

This study was supported by the Hanmi Group [0620140890 (2013-2607)], Seoul, Korea. The funder had no role in the design of the study; the collection, analysis, and interpretation of data; or in writing the manuscript.

\section{Availability of data and materials}

The dataset generated and analyzed during this study is available from the National Health Insurance Sharing Service. However, the authors have no right to share or provide these data. Information on how to request these data is available at https://nhiss.nhis.or.kr/bd/ab/bdaba021eng.do. The details and costs of the database are described at https://nhiss.nhis.or.kr/bd/ab/ bdaba022eng.do. To request the database, available only in Korean, visit https://nhiss.nhis.or.kr/bd/ay/bdaya001iv.do.

The questionnaire used in this study is not available in English. A Korean version of the questionnaire can be downloaded from.
http://www.law.go.kr/admRulLsInfoP.do?chrClsCd=\&admRulSeq=22 00000012541\#AJAX.

\section{Ethics approval and consent to participate}

This study protocol was approved by the Institutional Review Board of Seoul National University Hospital (IRB No. E-1703-020-836). Subjects were informed of the purpose of research and those participating in the NSPTA provided written informed consent, stating that these subjects understood that the information gathered through the NSPTA would be used for research purposes.

Administrative permission to access the NHID was acquired through the National Health Insurance Sharing Service (NHIS-2018-2-201).

Consent for publication

Not applicable.

\section{Competing interests}

The authors declare that they have no competing interests.

\section{Author details}

'Department of Family Medicine, Asan Medical Center, 88 Olympic-ro 43 gil, Songpa-gu, Seoul 05505, South Korea. ${ }^{2}$ Department of Family Medicine/ Supportive Care Center, Samsung Medical Center, School of Medicine, Sungkyunkwan University, Seoul, South Korea. ${ }^{3}$ Center for Clinical Epidemiology, Samsung Advanced Institute for Health Sciences \& Technology (SAIHST), Sungkyunkwan University, Seoul, South Korea. ${ }^{4}$ Department of Family Medicine, CHA Bundang Medical Center, CHA University, Seongnam-si, Gyeonggi-do, South Korea. ${ }^{5}$ Department of Family Medicine, Bumin Hospital, Seoul, South Korea. ${ }^{6}$ Health Promotion Center, Seoul National University Hospital, Seoul, South Korea. ${ }^{7}$ Department of Family Medicine, Seoul National University Hospital, Seoul, South Korea. ${ }^{8}$ Institute on Aging, Seoul National University College of Medicine, Seoul, South Korea.

Received: 22 April 2020 Accepted: 9 November 2020

Published online: 13 November 2020

\section{References}

1. Woodman R, Ferrucci L, Guralnik J. Anemia in older adults. Curr Opin Hematol. 2005;12(2):123-8.

2. Ble A, Fink JC, Woodman RC, Klausner MA, Windham BG, Guralnik JM, et al. Renal function, erythropoietin, and anemia of older persons: the InCHIANTI study. Arch Intern Med. 2005;165(19):2222-7.

3. Juárez-Cedillo T, Basurto-Acevedo L, Vega-García S, Manuel-Apolinar L, CruzTesoro E, Rodríquez-Pérez JM, et al. Prevalence of anemia and its impact on the state of frailty in elderly people living in the community: SADEM study. Ann Hematol. 2014:93(12):2057-62.

4. Thein M, Ershler WB, Artz AS, Tecson J, Robinson BE, Rothstein G, et al. Diminished quality of life and physical function in community-dwelling elderly with anemia. Medicine. 2009;88(2):107.

5. Agnihotri P, Telfer M, Butt Z, Jella A, Cella D, Kozma CM, et al. Chronic anemia and fatigue in elderly patients: results of a randomized, doubleblind, placebo-controlled, crossover exploratory study with epoetin alfa. J Am Geriatr Soc. 2007;55(10):1557-65.

6. Thaler-Kall K, Döring A, Peters A, Thorand B, Grill E, Koenig W, et al. Association between anemia and falls in community-dwelling older people: cross-sectional results from the KORA-age study. BMC Geriatr. 2014;14(1):29.

7. Bowling CB, Brown CJ, Allman RM, Warriner AH, Curtis JR, Warnock DG, et al. Low hemoglobin levels and recurrent falls in U.S. men and women: prospective findings from the REasons for geographic and racial differences in stroke (REGARDS) cohort. Am J Med Sci. 2013;345(6):446-54.

8. Artz AS. Anemia and the frail elderly. Seminars in hematology; 2008. Elsevier.

9. Bang S-M, Lee J-O, Kim YJ, Lee K-W, Lim S, Kim JH, et al. Anemia and activities of daily living in the Korean urban elderly population: results from the Korean longitudinal study on health and aging (KLoSHA). Ann Hematol. 2013;92(1):59-65.

10. Onem Y, Terekeci H, Kucukardali Y, Sahan B, Solmazgül E, Senol MG, et al. Albumin, hemoglobin, body mass index, cognitive and functional performance in elderly persons living in nursing homes. Arch Gerontol Geriatr. 2010;50(1):56-9. 
11. Röhrig G, Becker I, Schulz R-J, Lenzen-Großimlinghaus R, Willschrei P, Gebaue $\mathrm{S}$, et al. Association between hematologic parameters and functional impairment among geriatric inpatients: data of a prospective cross-sectional multicenter study ("GeriPrävalenz2013"). Maturitas. 2016;90:37-41.

12. Landi F, Russo A, Danese P, Liperoti R, Barillaro C, Bernabei R, et al. Anemia status, hemoglobin concentration, and mortality in nursing home older residents. J Am Med Dir Assoc. 2007:8(5):322-7.

13. Zakai NA, Katz R, Hirsch C, Shlipak MG, Chaves PH, Newman AB, et al. A prospective study of anemia status, hemoglobin concentration, and mortality in an elderly cohort: the cardiovascular health study. Arch Intern Med. 2005;165(19):2214-20.

14. Penninx BW, Guralnik JM, Onder G, Ferrucci L, Wallace RB, Pahor M. Anemia and decline in physical performance among older persons. Am J Med. 2003; 115(2):104-10

15. Penninx BW, Pahor M, Cesari M, Corsi AM, Woodman RC, Bandinelli S, et al. Anemia is associated with disability and decreased physical performance and muscle strength in the elderly. J Am Geriatr Soc. 2004;52(5):719-24.

16. Ramel A, Gudjonsson MC, Geirsdottir O, Thorsdottir I, Jonsson P. Hemoglobin and physical function after exercise intervention among community dwelling older adults. Innov Aging. 2017;1(Suppl 1):1052.

17. Zilinski J, Zillmann R, Becker I, Benzing T, Schulz R-J, Roehrig G. Prevalence of anemia among elderly inpatients and its association with multidimensional loss of function. Ann Hematol. 2014;93(10):1645-54.

18. Podsiadlo D, Richardson S. The timed "up \& go": a test of basic functional mobility for frail elderly persons. J Am Geriatr Soc. 1991;39(2):142-8.

19. Chun $\mathrm{SH}$, Cho B, Yang H-K, Ahn E, Han MK, Oh B, et al. Performance on physical function tests and the risk of fractures and admissions: findings from a national health screening of 557,648 community-dwelling older adults. Arch Gerontol Geriatr. 2017;68:174-80.

20. Donoghue OA, Savva GM, Cronin H, Kenny RA, Horgan NF. Using timed up and go and usual gait speed to predict incident disability in daily activities among community-dwelling adults aged 65 and older. Arch Phys Med Rehabil. 2014;95(10):1954-61.

21. Ekström H, Dahlin-Ivanoff S, Elmståhl S. Effects of walking speed and results of timed get-up-and-go tests on quality of life and social participation in elderly individuals with a history of osteoporosis-related fractures. J Aging Health. 2011;23(8):1379-99.

22. Huisman MG, Van Leeuwen BL, Ugolini G, Montroni I, Spiliotis J, Stabilini C, et al. "Timed up \& go": a screening tool for predicting 30-day morbidity in onco-geriatric surgical patients? A multicenter cohort study. PLoS One. 2014;9(1):1.

23. Wennie Huang WN, Perera S, VanSwearingen J, Studenski S. Performance measures predict onset of activity of daily living difficulty in communitydwelling older adults. J Am Geriatr Soc. 2010;58(5):844-52.

24. Kamel MA, Ebeid SM, Elakkad RM, Shafeek NE. Prevalence of anemia among community dwelling elderly in Dakahlia as a representative of rural areas in Egypt and its impact on their functional status. Adv Aging Res. 2015;4(05):168

25. Jans $\varnothing$, Bandholm T, Kurbegovic S, Solgaard S, Kjærsgaard-Andersen $P$ Johansson PI, et al. Postoperative anemia and early functional outcomes after fast-track hip arthroplasty: a prospective cohort study. Transfusion. 2016;56(4):917-25.

26. Cheol Seong S, Kim YY, Khang YH, Heon Park J, Kang HJ, Lee H, et al. Data resource profile: the national health information database of the National Health Insurance Service in South Korea. Int J Epidemiol. 2017:46(3):799-800.

27. Kim HS, Shin DW, Lee WC, Kim YT, Cho B. National screening program for transitional ages in Korea: a new screening for strengthening primary prevention and follow-up care. J Korean Med Sci. 2012;27(Suppl):S70-5.

28. World Health Organization. Nutritional anaemias: report of a WHO scientific group; 1968

29. Son KY, Shin DW, Lee JE, Kim SH, Yun JM, Cho B. Association of timed up and go test outcomes with future incidence of cardiovascular disease and mortality in adults aged 66 years: Korean national representative longitudinal study over 5.7 years. BMC Geriatr. 2020;20(1):1-9.

30. Son KY, Shin DW, Yang HK, Yun JM, Chun SH, Lee JK, et al. Effect of onetime brief additional counseling on periodic health examination for 40-and 66-year-olds: 2-year follow up of 101260 participants. Geriatr Gerontol Int. 2018;18(2):329-37.

31. Yang DW, Chey JY, Kim SY, Kim BS. The development and validation of Korean dementia screening questionnaire (KDSQ). J Korean Neurol Assoc. 2002;20(2):135-41.
32. Won CW, Yang KY, Rho YG, Kim SY, Lee EJ, Yoon JL, et al. The development of Korean activities of daily living (K-ADL) and Korean instrumental activities of daily living (K-IADL) scale. J Korean Geriatr Soc. 2002;6(2):107.

33. Cho MJ, Bae JN, Suh GH, Hahm BJ, Kim JK, Woo LD, et al. Validation of geriatric depression scale, Korean version (GDS) in the assessment of DSMIII-R major depression. J Korean Neuropsychiatr Assoc. 1999;38(1):48-63.

34. Chaves PH, Ashar B, Guralnik JM, Fried LP. Looking at the relationship between hemoglobin concentration and prevalent mobility difficulty in older women. Should the criteria currently used to define anemia in older people be reevaluated? J Am Geriatr Soc. 2002;50(7):1257-64.

35. Ferrucci L, Harris TB, Guralnik JM, Tracy RP, Corti MC, Cohen HJ, et al. Serum IL-6 level and the development of disability in older persons. J Am Geriatr Soc. 1999;47(6):639-46.

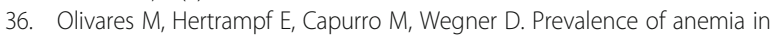
elderly subjects living at home: role of micronutrient deficiency and inflammation. Eur J Clin Nutr. 2000;54(11):834-9.

37. Kuipers H, Moran J, Mitchell DW, Shobe J, Dubravcic-Simunjak S, Sakai H, et al. Hemoglobin levels and athletic performance in elite speed skaters during the olympic season 2006. Clin J Sport Med. 2007;17(2):135-9.

38. Hopstock LA, Utne EB, Horsch A, Skjelbakken T. The association between anemia and falls in community-living women and men aged 65 years and older from the fifth Tromsø study 2001-02: a replication study. BMC Geriatr. 2017;17(1):292.

\section{Publisher's Note}

Springer Nature remains neutral with regard to jurisdictional claims in published maps and institutional affiliations.
Ready to submit your research? Choose BMC and benefit from:

- fast, convenient online submission

- thorough peer review by experienced researchers in your field

- rapid publication on acceptance

- support for research data, including large and complex data types

- gold Open Access which fosters wider collaboration and increased citations

- maximum visibility for your research: over $100 \mathrm{M}$ website views per year

At BMC, research is always in progress.

Learn more biomedcentral.com/submissions 